\title{
Abuso de Internet y adolescentes: gratificaciones, supervisión familiar y uso responsable. Implicaciones educativas y familiares.
}

\author{
Ana Cebollero Salinas \\ anacebollero@unizar.es \\ Universidad de Zaragoza, España \\ Jacobo Cano Escoriaza \\ jcano@unizar.es \\ Universidad de Zaragoza, España \\ Santos Orejudo Hernández \\ sorejudo@unizar.es \\ Universidad de Zaragoza, España
}

\begin{abstract}
Resumen
El abuso de Internet se ha convertido en un problema social de la adolescencia. Investigaciones anteriores sugieren que está relacionado con las gratificaciones que reciben al utilizarlo. Sin embargo, se desconoce si conjuntamente el uso responsable (netiqueta) y la supervisión familiar pueden equilibrar las gratificaciones que promueven este riesgo. Por ello, el objetivo de este trabajo es analizar en qué medida las gratificaciones, la supervisión familiar y su uso responsable predicen el abuso de Internet y con ello comprender mejor este riesgo en la adolescencia de cara a que docentes y familias puedan establecer estrategias para su prevención según el género y edad. En el estudio participaron 555 adolescentes (55.7\% chicos) de 10-40 ESO (M: 13. 97 años y SD: 1. 27). Los datos se obtuvieron través de cuestionarios. Los resultados revelan que, aunque principalmente el entretenimiento en chicos y la evasión en chicas promueven el abuso, educar la netiqueta es un factor de protección importante para ambos géneros y todas las edades, así como la supervisión familiar para las chicas y para los adolescentes de 12-13 años. Se discuten las implicaciones educativas y familiares.
\end{abstract}

\section{Palabras claves}

Abuso de Internet, Redes sociales, Usos y Gratificaciones, Netiqueta, Mediación familiar, Educación crítica 


\title{
Internet Abuse and teenagers: Gratifications, Family Supervision, and Responsible Use. Educational and family implications.
}

\author{
Ana Cebollero Salinas \\ anacebollero@unizar.es \\ Universidad de Zaragoza \\ Jacobo Cano Escoriaza \\ jcano@unizar.es \\ Universidad de Zaragoza \\ Santos Orejudo Hernández \\ sorejudo@unizar.es \\ Universidad de Zaragoza
}

\begin{abstract}
Internet abuse has become a social problem in adolescence. Previous research suggested that it is related to the gratifications they receive from using it. However, it is unknown whether continued responsible use (netiquette) and family supervision can balance the rewards that promote this risk. Thus, the purpose of this paper is to analyze the extent to which gratifications, family supervision and responsible use predict Internet abuse and thereby better understand this risk in adolescence so that teachers and families can establish strategies for its prevention according to the gender and age. 555 adolescents ( $55.7 \%$ boys) from 1st to 4th year of ESO (M: 13.97 years old and SD: 1.27 ) participated in the study. The data were obtained through questionnaires. The results reveal that, although mainly entertainment in boys and avoidance in girls promote abuse, educating netiquette is an important protection factor for both genders and all ages, as well as family supervision in girls and boys. in adolescents 12-13 years old. Educational and family implications are discussed.
\end{abstract}

\section{Keywords}

Internet abuse, Social network sites, Uses and gratifications, Netiquette, Parental mediation, Critical education 


\section{Introducción}

La tecnología se ha convertido en un recurso básico para la vida diaria facilitando la comunicación, el aprendizaje, el entretenimiento, así como el desarrollo laboral y económico. En España, el Instituto Nacional de Estadística señala que el 94,5\% de los menores de 10-15 años son usuarios de Internet (INE, 2020). Este uso extensivo de Internet conlleva la aparición de nuevas conductas y hábitos que despiertan la inquietud de las familias y centros educativos pues, a pesar de sus numerosos beneficios, llevan asociados al mismo tiempo algunos riesgos como la dependencia a Internet (Giménez et al., 2017).

La adicción a Internet no está establecida como un trastorno adictivo en el DSM-V lo cual no significa que no sea negativo. De ahí que la tendencia dominante sea utilizar términos como abuso, uso desadaptativo, uso problemático, para referirse a un patrón que incluye la necesidad recurrente de conectarse a Internet, deficiencias en la autorregulación, el uso como recurso de escapar de los problemas, y la aparición de consecuencias negativas en la vida cotidiana como el reemplazo de las relaciones familiares y sociales y cambios en los hábitos de salud (Caplan, 2010). En este artículo son términos que utilizaremos indistintamente.

Diversos factores de riesgo se relacionan con el abuso de Internet como los rasgos de personalidad correlacionando positivamente con la responsabilidad y negativamente con la extraversión y la estabilidad emocional (Marino et al., 2016). Otros estudios aportan evidencias de su relación con altos niveles de diversión y entretenimiento y ser chica (Malo-Cerrato et al., 2018), con estados de ánimo depresivo, de tristeza e ira y con el estrés asociado en las relaciones sociales y las obligaciones con el trabajo (Li et al., 2015). Análisis realizados en diferentes países y culturas demuestran que el uso persistente de Internet aumenta el riesgo (Müller et al., 2017; Rial et al., 2014) sin embargo es muy probable que el uso prolongado por sí mismo no implique síntomas desadaptativos (Griffiths, 2010).

La prevalencia global media adolescentes varía entre el $5 \%$ y el $15,2 \%$ en Europa y entre el 2,5\% y $26,8 \%$ en países asiáticos (Kuss et al., 2014; Wang et al., 2016). En España, un $21.3 \%$ de los adolescentes están en riesgo de desarrollar conductas adictivas a Internet debido al uso abusivo de las redes sociales (Fundación Mapfre, 2014). Un estudio reciente con población adolescente destaca que casi la mitad de los estudiantes realiza un uso desadaptado o tiene indicios de abuso de Internet (Díaz-López et al., 2020) alertando a educadores y familias por las problemáticas que acarrea como en la autoestima y las bajas aspiraciones académicas (Mo et al., 2020), depresión e ideación suicida (Kaess et al., 2014) y un aumento de situaciones de ciberbullying (Yudes-Gómez et al., 2018). La prevalencia y sus impactos negativos hace que sea necesario continuar investigando sobre este comportamiento. En concreto, en el presente artículo queremos combinar varios predictores del abuso de Internet que rara vez se han investigado conjuntamente con el objetivo de arrojar luz sobre cómo la supervisión familiar y educar en el uso responsable de Internet pueden equilibrar las gratificaciones que los adolescentes buscan al usar Internet derivando en un uso desadaptativo.

\section{a. Supervisión familiar}

La familia es el primer grupo social donde los menores adquieren costumbres, valores y estilos de vida. La incursión de la tecnología genera nuevas dinámicas familiares que deben ser estudiadas. En este trabajo asumimos como supervisión familiar las diferentes interacciones o mediaciones que se establecen entre padres e hijos en torno al uso y consumo de los medios (Sasson \& Mesch, 2019).

Para reducir los riesgos, de los dos principales tipos de mediación, la mediación activa que supone dialogar, acompañar y orientar a los hijos sobre las actividades en línea, funciona mejor que la 
restrictiva -establecimiento de normas y límites en el tiempo y contenido de Internet- (Shin \& Kang, 2016). Algunos autores resaltan que también la mediación restrictiva lo consigue, aunque indirectamente al limitar el acceso (Khurana et al., 2015) pero lo hace a costa de reducir las oportunidades que ofrece Internet (Livingstone et al., 2017).

Según un reciente estudio a nivel europeo con población entre 9 y 17 años, el grado de supervisión familiar varía de unos países a otros, así como el tipo de supervisión familiar que se lleva a cabo. Los países con mediaciones más restrictivas son Alemania y Francia, quienes controlan el uso de las redes sociales con porcentajes en torno al 30\% en comparación a Italia (21\%) y España (17\%). Este tipo de mediación es similar para ambos géneros y decrece con la edad. Por otro lado, los países donde el $50 \%$ de los niños reflejan que nunca o casi nunca hablan con sus padres de lo que hacen en Internet son junto a España, Suiza, República Checa y Polonia. En contraposición al 17\% de Francia o el 19\% de Alemania siendo mayoritarios los países que les dedican más tiempo a las chicas y a los más jóvenes (Smahel et al., 2020). Respecto a España estos datos son confirmados por otros estudios como Díaz-López et al., (2020) para adolescentes entre 11 y 18 años, señalando que el $56 \%$ afirma utilizar Internet sin supervisión de sus padres apreciándose una disminución de la misma a medida que aumenta la edad y siendo mayor en las chicas. También en países de Iberoamérica como Chile los datos indican niveles similares a España, donde el 58\% navegan solos y el 36,7\% lo hace sin límite de tiempo (Berríos et al., 2015). Como algunos autores evidencian parece ser que la implementación de la supervisión parental es mayor cuando los padres tienen una mayor percepción del riesgo y de la eficacia de la mediación (Chang et al., 2019) tendiendo a ser más negativa y reactiva que positiva y proactiva (Bartau-Rojas et al., 2018).

No cabe duda que la supervisión familiar tiene un efecto regulador directo sobre la protección de riesgos de Internet. Se ha evidenciado su papel en la salvaguarda de los perfiles de ciberagresor o cibervíctima (Baldry et al., 2019), de abusar de Internet (Kalmus et al., 2015) y de los smartphones (Chang et al., 2019). En este sentido, se constata que los menores que son menos supervisados tienen mayores niveles de conductas de aislamiento con la tecnología (Giménez et al., 2017) por lo que su estudio puede promocionar un uso saludable de Internet muy amplio.

\section{b. Netiqueta o uso responsable de Internet}

En la adolescencia impera un uso de Internet comunicativo, social y de entretenimiento entre los adolescentes (Smahel et al., 2020). Además, se ha encontrado que adolescentes con un uso problemático de Internet tienden a incurrir en actitudes de violencia como el ciberacoso (YudesGómez et al., 2018) y suelen tener múltiples comportamientos de riesgo (Durkee et al., 2016). Para hacer frente a conductas agresivas, y de manera asociada al abuso de Internet, los investigadores sugieren constantemente educar a los adolescentes en netiqueta o uso responsable de Internet (Ang et al., 2014; Ortega et al., 2012; Park et al., 2014) refiriéndose al conjunto de reglas y pautas para usar Internet de manera adecuada (Shea, 1994) como por ejemplo solicitar permiso para usar información personal de otros y responder de forma calmada al sentirse agredido en la comunicación virtual.

En la literatura científica encontramos numerosos estudios que plantean la necesidad de educar en el uso responsable en relación al abuso de Internet (Díaz et al., 2020; Malo-Cerrato et al., 2018; Rodríguez-Gómez et al., 2018; entre otros) sin embargo, hasta la fecha no existen estudios que analicen su relación excepto, Ortega et al., (2012) que encuentra relaciones inversas con la dependencia a Internet y la intensidad de uso de las redes sociales y Park et al., (2014) con el tiempo de uso de Internet y el ciberbullying. 


\section{c. Usos y Gratificaciones de Internet}

La teoría de los usos y gratificaciones fue concebida para tratar de responder al interrogante de por qué las personas se exponen a los distintos medios tradicionales de comunicación de masas como la radio, televisión, cine, y el periódico, considerando que cada mass media proporciona una combinación única de características que lo hace adecuado para la satisfacción de cierta necesidad y los usuarios utilizan motivos que se adaptan de acuerdo a los resultados de su uso (Katz et al., 1973). En esta teoría se considera que los individuos son activos y permite medir las acciones cognitivas (información, conocimiento y comprensión), afectivas (emocional, placentera y estética), integradoras (mejora de la credibilidad, estabilidad y estatus), de integración social (fortaleciendo vínculos familiares, amigos y mundo) y necesidades de escape, que son producidas como efecto secundario a una adhesión tecnológica (Katz, et al., 1974).

Numerosos autores defienden el uso de esta aproximación teórica como marco adecuado para aplicarla a la diversidad de medios y tecnologías de la información y comunicación como Internet (Papacharissi \& Rubin, 2000), las redes sociales como Facebook (Masur et al., 2014; Ryan et al., 2014), los videojuegos (González-Vázquez \& Igartua, 2018; Sherry et al., 2006). Como resultado de ello, algunas necesidades han sido matizadas y otras especificadas para los nuevos medios explicando así el comportamiento de los usuarios (Igartua \& Rodríguez-de-Dios, 2016). Por ejemplo, en el caso de las redes sociales la autopresentación online o ser bien considerado por otros en línea, el logro virtual demostrando la influencia que se tiene en las redes sociales pueden satisfacer las necesidades integradoras, así como el juego online satisface las necesidades de escape y afectivas del usuario (Heravi et al., 2018).

Estudios previos al analizar las gratificaciones asociadas al uso de las redes sociales han identificado que el entretenimiento (Park \& Lee, 2014), mantenimiento de relaciones, (Papacharissi, Z., \& Mendelsohn, 2011; Park \& Lee, 2014), autoexpresión (Park \& Lee, 2014), comunicación (Park \& Lee, 2014), autopresentación y escapismo (Papacharissi, \& Mendelsohn, 2011), ocupan un lugar destacado entre los motivos identificados.

Refiriéndose a este razonamiento algunos estudios han investigado qué tipo de gratificaciones predicen el abuso de Internet. Así Kim \& Haridakis, (2009) en estudiantes de pregrado de EE.UU destacan el entretenimiento, la emoción y el escapismo como principales motivaciones. Más concretamente un reciente meta-análisis que incluye 56 estudios sobre el uso problemático de Facebook concluye que las dos principales razones que se asocian a este riesgo son usar las redes sociales para reducir los estados de ánimo negativos y satisfacer necesidades emocionales internas como pasar el tiempo (Marino et al., 2018). Otros estudios plantean la autopresentación, el escapismo y la construcción de relaciones sociales como principales satisfacciones buscadas (Chen \& Kim, 2013), obtener mayor presencia social y velar por la propia imagen (García-Ruiz et al., 2018). Estos hallazgos sugieren que las motivaciones de uso pueden ser cruciales para explicar el abuso de Internet.

Con estos hallazgos podemos hipotetizar que, si los adolescentes con uso desaptativo en Internet buscan en gran medida presencia social, buena imagen y logro en las relaciones sociales en Internet, el que se relacionen de manera respetuosa y responsable al interactuar en la Red puede actuar de papel protector. Junto a ello también la supervisión familiar. Sin embargo, hasta la actualidad no hay estudios que analicen estos factores conjuntamente. Por tanto, los objetivos de este trabajo serán 1) analizar las relaciones entre el abuso de Internet y las variables presentadas y 2) evaluar qué variables predicen el abuso de Internet diferenciando el género y la edad. De esta forma podremos establecer estrategias efectivas, escolares y familiares, que mejoren la prevención de este riesgo. 


\section{Método}

\section{a. Participantes}

Participaron 555 adolescentes con edades comprendidas entre los 12 y 17 años (M=13.97; DT $=1.27$ ), de los cuales el $55,7 \%$ fueron chicas y el 44,3\% chicos que estaban cursando Educación Secundaria Obligatoria 10 ESO $(24.7 \%)$, 20 ESO $(20.5 \%), 30$ ESO $(25.4 \%)$ y 40 ESO $(29.4 \%)$ de Aragón (España). Se realizó un muestreo no probabilístico incidental en el que se seleccionó la muestra por accesibilidad a los centros educativos.

\section{b. Instrumentos}

\section{Escala de usos y gratificaciones}

Esta escala consta de 18 ítems, correspondientes a cinco gratificaciones del uso de Internet validada por García-Ruiz et al., (2018) que son: Evasión (por ejemplo: "Uso Internet cuando me siento frustrado" ( $a=.84$ ), Entretenimiento (por ejemplo: "Uso Internet porque es entretenido" (a $=.85$ ), Presencia social (por ejemplo: "Me siento conectado a otras personas" ( $a=.80$ ), Autopresentación (por ejemplo: "En Internet quiero que otras personas me perciban como agradable" $(a=.90)$, y Logro Uso (por ejemplo: "Internet para demostrar a otros que soy el mejor" $(a=.55)$. Se aplica en una escala tipo Likert con valores que van desde $1=$ Totalmente en desacuerdo hasta $5=$ Totalmente de acuerdo.

\section{Uso responsable de Internet}

La subesala de uso responsable forma parte del Cuestionario para la evaluación de la calidad dela ciberconducta EsCaCiber (Ortega et al., 2012). La escala total consta de 30 ítems siendo su consistencia interna .76 y la subescala utilizada tiene 4 ítems, siendo su alpha de Cronbach .70. Está dirigido a edades entre 10-19 años. Se trata de una escala Likert de frecuencia de 5 puntos de 0 =nunca a 4 =siempre. Donde los ítems hacen referencia a la valoración de respeto (por ejemplo "Me dirijo a los demás con respeto en las redes sociales", al cumplimiento de la netiqueta (por ejemplo "Cuando publico algo de una persona le pido permiso"), a actuar pensando antes en las consecuencias (por ejemplo "Antes de comentar o criticar sobre alguien en las redes sociales pienso en el daño que puedo hacerle") y con autocontrol (por ejemplo "Si me siento agredido en las redes sociales, trato de responder de forma calmada y no violenta").

\section{Supervisión familiar}

Utilizamos para la medida la subescala Supervisión familiar del Cuestionario para la evaluación de la calidad de la ciberconducta EsCaCiber (Ortega et al., 2012) que valora el control y ayuda familiar en las actividades en redes sociales que la familia realiza con sus hijos e hijas como por ejemplo "Mis padres me ayudan a hacer un uso adecuado de las Redes Sociales". La subescala utilizada tiene 4 ítems, siendo su alpha de Cronbach .77. Se trata de una escala Likert de frecuencia de 5 puntos de $0=$ nunca a 4 =siempre.

\section{Abuso de Internet}

El cuestionario de Experiencias relacionadas con Internet (CERI) (Casas et al., 2013) cuantifica la adicción a la Red contemplando dos factores: la dimensión interpersonal relacionado con el uso abusivo de Internet y redes sociales (por ejemplo "¿Te parece más fácil relacionarse con las personas a través de Internet que cara a cara?") y la dimensión intrapersonal de su uso (por 
ejemplo "¿Cuándo estás en problemas, ¿conectarte a Internet te ayuda a escapar de ellos?"). Los ítems se distribuyen en una escala Likert de 4 puntos que van de 1 (nunca) a 4 (mucho). Los índices de fiabilidad alcanzaron a interpersonal $=.70$, $a$ intrapersonal $=.79$ y total $a=.86$.

\section{c. Procedimiento}

En primer lugar, se solicitó a los centros educativos su participación para lo cual se les remitió un breve informe sobre los objetivos y alcance de la investigación. Tras recibir el visto bueno por parte de los equipos directivos y recabar los consentimientos informados de los padres, se pasaron las encuestas al alumnado. El horario convenido fue dentro del lectivo con la presencia de un investigador y una duración de 30 minutos. A continuación, se procedió al análisis de los cuestionarios, al vaciado y análisis de la información con el programa estadístico SPSS versión 26.0.

La investigación se desarrolló de acuerdo a los estándares éticos y fue aprobada por el Comité Coordinador de Ética de la Investigación Biomédica de Aragón.

\section{Procedimiento estadístico}

Se obtienen estadísticos descriptivos para establecer las características sociodemográficas. y psicológicas de los participantes. Las relaciones entre las variables de estudio fueron analizadas utilizando correlaciones bivariadas. Se calcula el índice de fiabilidad y validez de los instrumentos utilizados a través del alfa de Cronbach. Se realizan análisis correlacionales. Se explora la capacidad predictiva del abuso de Internet respecto al resto de variables contempladas mediante análisis de regresión lineal múltiple. Este procedimiento se hace, por un lado, con grupos de chicos y chicas, y por otro, con grupos de edad (12-13 años vs. 14 o más). Para realizar esta regresión, se utiliza un método de inclusión por bloques de los grupos de variables: bloque 1: supervisión familiar; bloque 2: uso responsable y bloque 3: gratificaciones. Para analizar la mejora de los diferentes bloques y valorar el aporte de nuevas variables, se usa el método Steep-wise dentro de cada bloque. Se tienen en cuenta el cumplimiento de los supuestos de la regresión múltiple (Pardo y Ruiz, 2007). Para ello se utiliza el paquete estadístico SPSS 22.0.

\section{Resultados}

\section{a. Relación entre las variables de estudio según el género y la edad}

En la Tabla 1 se muestran los análisis descriptivos de toda la muestra y las correlaciones entre las distintas variables según el género.

Tanto en las chicas como en los chicos el abuso de Internet se correlaciona positivamente con todos los tipos de gratificaciones de uso y negativamente con el uso responsable y supervisión familiar. Sin embargo, en el caso de esta última sólo se correlaciona significativamente en el caso de las chicas. En estas relaciones hay diferencias en las puntuaciones alcanzadas, siendo mayores para las chicas entre abuso de internet y uso responsable $(-.356$ vs -.260$)$ y con evasión (.535 vs $.480)$. Sin embargo, en los chicos son mayores las relaciones entre el abuso de internet y el entretenimiento (.541 vs .470), el logro (.446 vs .339), la autopresencia (.349 vs 275) y la presencia social (.398 vs 328$)$.

Las gratificaciones también correlacionan entre sí con puntuaciones por encima de .30, destacando la relación entre evasión y entretenimiento (.643 en chicos y .629 en chicas), entretenimiento con 
autopresentación (.618 en chicos y .380 en chicas) y entretenimiento con presencia social (.636 en chicos vs .557 en chicas).

\begin{tabular}{|c|c|c|c|c|c|c|c|c|c|c|c|c|}
\hline & Media & DT & 1 & 2 & 3 & 4 & 5 & 6 & 7 & 8 & $\begin{array}{l}\text { Medi } \\
\text { a }\end{array}$ & DT \\
\hline \multicolumn{11}{|c|}{ Chicos } & & \\
\hline $\begin{array}{l}\text { 1. Supervisión } \\
\text { familiar }\end{array}$ & 7.45 & 4.137 & & $\begin{array}{l}.204 * \\
*\end{array}$ & .020 & .072 & .046 & .043 & $.142 *$ & -.105 & 6.18 & 4.077 \\
\hline $\begin{array}{l}\text { 2. Uso } \\
\text { responsable }\end{array}$ & 11.99 & 3.497 & $\begin{array}{l}.264 * \\
*\end{array}$ & & .016 & -.056 & -.120 & .127* & .065 & $\begin{array}{l}-.260 \\
* *\end{array}$ & \begin{tabular}{l|}
11.2 \\
2
\end{tabular} & 3.908 \\
\hline $\begin{array}{l}3 . \\
\text { Entretenimien } \\
\text { to } 1\end{array}$ & 19,36 & 5.048 & -124 & $\begin{array}{l}.136 \\
* *\end{array}$ & & $\begin{array}{l}.643 * \\
*\end{array}$ & $\begin{array}{l}.454 * \\
*\end{array}$ & $\begin{array}{l}.618^{*} \\
*\end{array}$ & $\begin{array}{l}.636 * \\
*\end{array}$ & $\begin{array}{l}.541 * \\
*\end{array}$ & \begin{tabular}{|l|}
18.2 \\
0
\end{tabular} & 5.562 \\
\hline 4. Evasión & 10.08 & 3.935 & $\begin{array}{l}-.176 \\
* *\end{array}$ & $\begin{array}{l}.186 \\
* *\end{array}$ & $\begin{array}{l}.629 * \\
*\end{array}$ & & $\begin{array}{l}.463 * \\
*\end{array}$ & $\begin{array}{l}.385 * \\
*\end{array}$ & $\begin{array}{l}.438^{*} \\
*\end{array}$ & $\begin{array}{l}.480 * \\
*\end{array}$ & 9.60 & 4.032 \\
\hline 5. Logro & 3.54 & 1.831 & -.059 & $\begin{array}{l}-.178 \\
* *\end{array}$ & $\begin{array}{l}.325 * \\
*\end{array}$ & $\begin{array}{l}.384 * \\
*\end{array}$ & & $\begin{array}{l}.356 * \\
*\end{array}$ & $\begin{array}{l}.374 * \\
*\end{array}$ & $\begin{array}{l}.446 * \\
*\end{array}$ & 3.94 & 1.977 \\
\hline $\begin{array}{l}6 . \\
\text { Autopresencia }\end{array}$ & 6.06 & 2.221 & -.013 & -.056 & $\begin{array}{l}.380 * \\
*\end{array}$ & $\begin{array}{l}.278 * \\
*\end{array}$ & $\begin{array}{l}.300 * \\
*\end{array}$ & & $\begin{array}{l}.635 * \\
*\end{array}$ & $\begin{array}{l}.349 * \\
*\end{array}$ & 5.86 & 2.228 \\
\hline $\begin{array}{l}\text { 7. Presencia } \\
\text { social }\end{array}$ & 12.53 & 3.827 & -.052 & $\begin{array}{l}-.141 \\
*\end{array}$ & $\begin{array}{l}.557 * \\
*\end{array}$ & $\begin{array}{l}.368^{*} \\
*\end{array}$ & $\begin{array}{l}.247 * \\
*\end{array}$ & $\begin{array}{l}.428 * \\
*\end{array}$ & & $\begin{array}{l}.398 * \\
*\end{array}$ & \begin{tabular}{|l|l}
11.8 \\
0
\end{tabular} & 4.114 \\
\hline $\begin{array}{l}\text { 8. Abuso } \\
\text { Internet }\end{array}$ & 20.22 & 5.068 & $\begin{array}{l}-.216 \\
* *\end{array}$ & $\begin{array}{l}-.356 \\
* *\end{array}$ & $\begin{array}{l}.470 * \\
*\end{array}$ & $\begin{array}{l}.535^{*} \\
*\end{array}$ & $\begin{array}{l}.339 * \\
*\end{array}$ & $\begin{array}{l}.275^{*} \\
*\end{array}$ & $\begin{array}{l}.328 * \\
*\end{array}$ & & $\begin{array}{l}20.5 \\
4\end{array}$ & 5.091 \\
\hline & & & & & & C & & & & & & \\
\hline
\end{tabular}

Tabla 1. Correlaciones entre las distintas variables según el género

Nota: $* \mathrm{p}<.05 ; * * \mathrm{p}<.01$. 1Dimensiones de gratificaciones de uso de Internet

Por otro lado, la supervisión familiar se relaciona, en ambos casos, con el uso responsable siendo algo mayor en el caso de las chicas (.264 vs .204). Sin embargo, para los dos grupos las gratificaciones no están asociadas con la supervisión familiar -tan sólo con evasión en el caso femenino y con presencia social en el caso masculino- y en el caso de los chicos las gratificaciones tampoco lo hacen con el uso responsable excepto con autopresencia.

Para analizar las correlaciones según la edad hemos establecido dos grupos, los de menor edad (12 y 13 años, $42.1 \%$ de la muestra) y los mayores (14 años en adelante, $57.9 \%$ ). En la Tabla 2 se muestran las correlaciones entre variables según los bloques de edad.

En los dos grupos de edades resultaron significativas las relaciones entre abuso de Internet y todas las variables exceptuando en los más mayores, la supervisión familiar. La relación entre abuso de Internet y uso responsable alcanza puntuaciones mayores en el grupo de 14 años en adelante (-.351 vs -.206), sin embargo, son mayores las relaciones con las gratificaciones de uso para el grupo de 12-13 años excepto en la autopresencia (.292 -12-13 años- vs .319-14 en adelante-). Entre los adolescentes la relación entre el entretenimiento y la presencia social es mayor en el grupo de 14 años en adelante (.671 vs .549), así como con la evasión (.664 vs .612) y el logro (.468 vs .294). 


\begin{tabular}{|c|c|c|c|c|c|c|c|c|c|c|c|c|}
\hline & Media & DT & 1 & 2 & 3 & 4 & 5 & 6 & 7 & 8 & $\begin{array}{l}\text { Medi } \\
a\end{array}$ & DT \\
\hline \multicolumn{13}{|c|}{14 o más } \\
\hline $\begin{array}{l}1 . \\
\text { Supervisi } \\
\text { ón } \\
\text { familiar }\end{array}$ & 8.47 & \begin{tabular}{|l|}
4.02 \\
2
\end{tabular} & & $.189 * *$ & $\mid .057$ & .001 & .075 & -.008 & \begin{tabular}{|l|}
$.131 *$ \\
\end{tabular} & -.054 & 5.89 & 3.930 \\
\hline $\begin{array}{l}\text { 2. Uso } \\
\text { responsa } \\
\text { ble }\end{array}$ & 12.19 & $\begin{array}{l}3.76 \\
0\end{array}$ & $.277^{* *}$ & & -.020 & $\begin{array}{l}-.113 * \\
*\end{array}$ & $\begin{array}{l}-.113 * \\
*\end{array}$ & .001 & -.058 & $\begin{array}{l}-.351 \\
* *\end{array}$ & $\begin{array}{l}11.3 \\
2\end{array}$ & 3.630 \\
\hline $\begin{array}{l}3 . \\
\text { Entreteni } \\
\text { miento1 }\end{array}$ & 18.20 & $\begin{array}{l}5.52 \\
6\end{array}$ & -.110 & -.065 & & $.612 * *$ & $.294 * *$ & $\begin{array}{l}.499 * \\
*\end{array}$ & $\begin{array}{l}.549 * \\
*\end{array}$ & $\begin{array}{l}.436 * \\
*\end{array}$ & $\begin{array}{l}19.2 \\
5\end{array}$ & 5.135 \\
\hline $\begin{array}{l}4 . \\
\text { Evasión1 }\end{array}$ & 9.07 & $\begin{array}{l}3.94 \\
8\end{array}$ & -.023 & -.082 & $\begin{array}{l}.664 * \\
*\end{array}$ & & $\begin{array}{l}.347 \\
* *\end{array}$ & $\begin{array}{l}.354 * \\
*\end{array}$ & $\begin{array}{l}.394 * \\
*\end{array}$ & $\begin{array}{l}.469 * \\
*\end{array}$ & $\begin{array}{l}10.3 \\
7\end{array}$ & 3.927 \\
\hline 5. Logro1 & 3.37 & $\begin{array}{l}1.88 \\
6\end{array}$ & -.069 & $\begin{array}{l}-.193 * \\
*\end{array}$ & $\begin{array}{l}.468 * \\
*\end{array}$ & & & $\begin{array}{l}.317 * \\
*\end{array}$ & $\begin{array}{l}.245 * \\
*\end{array}$ & $\begin{array}{l}.290 * \\
*\end{array}$ & 3.94 & 1.887 \\
\hline $\begin{array}{l}6 . \\
\text { Autoprese } \\
\text { ncia1 }\end{array}$ & 5.91 & $\begin{array}{l}2.31 \\
2\end{array}$ & .077 & .092 & $\begin{array}{l}.483 * \\
*\end{array}$ & $.291 * *$ & $.322 * *$ & & $\begin{array}{l}.517^{*} \\
*\end{array}$ & $\begin{array}{l}.319 * \\
*\end{array}$ & 6.01 & 2.171 \\
\hline $\begin{array}{l}7 . \\
\text { Presencia } \\
\text { social1 }\end{array}$ & 12.05 & $\begin{array}{l}4.08 \\
2\end{array}$ & -.039 & .017 & $\begin{array}{l}.671 * \\
*\end{array}$ & $.534 * *$ & $.369 * *$ & $\begin{array}{l}.534 * \\
*\end{array}$ & & $\begin{array}{l}.323 * \\
*\end{array}$ & $\begin{array}{l}12.3 \\
0\end{array}$ & 3.900 \\
\hline $\begin{array}{l}\text { 8. Abuso } \\
\text { Internet }\end{array}$ & 18.94 & $\begin{array}{l}4.77 \\
2\end{array}$ & $\begin{array}{l}-.203 * \\
*\end{array}$ & $\begin{array}{l}-.206 * \\
*\end{array}$ & $\begin{array}{l}.575 * \\
*\end{array}$ & $.529 * *$ & $.505 * *$ & $\begin{array}{l}.292 * \\
*\end{array}$ & $\begin{array}{l}.415 * \\
*\end{array}$ & & $\begin{array}{l}21.2 \\
6\end{array}$ & 5.064 \\
\hline
\end{tabular}

Tabla 2. Correlaciones entre las distintas variables según los bloques de edad

Nota: $* \mathrm{p}<.05 ; * * \mathrm{p}<.01$. ${ }^{1}$ Dimensiones de gratificaciones de uso de Internet

\section{b. Variables que predicen el abuso a Internet según el género y edad.}

En las tablas 3 y 4 se muestran los resultados de las ecuaciones de regresión según el género. Para el caso de los chicos, el modelo explica el $41.2 \%$ de la variabilidad del abuso de Internet y el $35.9 \%$ para las chicas. No obstante, en cuanto a las variables, los resultados no son iguales. En el caso de las chicas, tal como muestra la tabla 3, los factores de protección ante el abuso de Internet son la supervisión familiar y el uso responsable, aportando un $14.3 \%$ de la variabilidad al modelo. Sin embargo, en el caso de los chicos (tabla 4) es el uso responsable el único factor protector ante el abuso de Internet. Respecto a los factores de riesgo, en el caso de las chicas, la gratificación de la evasión alcanza el mayor peso, explicando un $21.6 \%$, y ya muy por debajo el entretenimiento $(2.7 \%)$ y el logro (1\%) mientras que en el caso de los chicos la gratificación que más explica la variabilidad del abuso de Internet es el entretenimiento (29.7\%), seguido del logro $(3.5 \%)$ y de la evasión (1.2\%). 


\begin{tabular}{|l|c|c|c|c|c|c|c|c|}
\hline \multicolumn{1}{|c|}{ Chicas } \\
\hline & 1 & 2 & 3 & 4 & 5 & $R^{2}$ & $\Delta R^{2}$ & Cambio F \\
\hline $\begin{array}{l}\text { 1.Supervisión } \\
\text { Familiar }\end{array}$ & -.264 & -.161 & -.081 & -.079 & -.084 & .047 & .047 & $15.005^{* *}$ \\
\hline $\begin{array}{l}\text { 2.Uso } \\
\text { responsable }\end{array}$ & & -.466 & -.362 & -.357 & -.338 & .143 & .096 & $25.488^{* *}$ \\
\hline 3. Evasión1 & & & .615 & .466 & .406 & .359 & .216 & $56.920^{* *}$ \\
\hline $\begin{array}{l}\text { 4.Entretenimie } \\
\text { nto1 }\end{array}$ & & & & .211 & .197 & .386 & .027 & $47.712^{* *}$ \\
\hline 5. Logro 1 & & & & & .300 & .395 & .010 & $39.635^{* *}$ \\
\hline
\end{tabular}

Tabla 3. Análisis de regresión entre variables que miden el abuso de Internet en las chicas Nota: $* p<.05$. ** $p<.01$. 1. Dimensiones de gratificaciones de uso

\begin{tabular}{|l|c|c|c|c|c|c|c|}
\hline \multicolumn{7}{|c|}{ Chicos } \\
\hline & 1 & $\begin{array}{c}2 \\
\beta\end{array}$ & $\begin{array}{c}3 \\
\beta\end{array}$ & $\begin{array}{c}4 \\
\beta\end{array}$ & $R^{2}$ & $\Delta R^{2}$ & Cambio F \\
\hline $\begin{array}{l}\text { 1.Uso } \\
\text { responsable }\end{array}$ & -.339 & -.350 & -.315 & -.307 & .068 & .068 & $17.887^{* *}$ \\
\hline $\begin{array}{l}2 . \text { Entretenimiento } \\
1\end{array}$ & & .499 & .410 & .336 & .365 & .297 & $69.695^{* *}$ \\
\hline $\begin{array}{l}\text { 3. Logro 1 } \\
\text { 4. Evasión1 }\end{array}$ & & & .549 & .471 & .402 & .035 & $53.757^{* *}$ \\
\hline
\end{tabular}

Tabla 4. Análisis de regresión entre variables que miden el abuso de Internet en los chicos Nota: $* p<.05 . * * p<.01$. 1. Dimensiones de gratificaciones de uso

Los resultados de las ecuaciones de regresión realizadas según la edad (tablas 5 y 6 ) muestran que el modelo de los más jóvenes, $12-13$ años, explica un $44.8 \%$ de la variabilidad del abuso de Internet y el de los mayores un $36.4 \%$. Las variables que se van incorporando son distintas. En el caso de los jóvenes los factores de protección son la supervisión familiar y el uso responsable justificando un $6.5 \%$ de la variabilidad. En el caso de los mayores, sólo lo es el uso responsable pero aumentado al $12.3 \%$ su explicación. También sucede en el caso de los factores de riesgo donde, en el caso de los adolescentes de 12-13 años la gratificación del entretenimiento aporta un $30.4 \%$ a la variabilidad y a medida que se incorporan otras gratificaciones su coeficiente pierde peso. El logro y la evasión aportan un $5.9 \%$ y $2 \%$ respectivamente. Para los chicos y chicas de 14 años en adelante es la evasión la gratificación que más aporta (18.7\%) seguido del entretenimiento $(4.3 \%)$ y de la autopresentación $(1.1 \%)$. 


\section{2-13 años}

\begin{tabular}{|l|c|c|c|c|c|c|c|c|} 
& 1 & 2 & 3 & 4 & 5 & $R^{2}$ & $\Delta R^{2}$ & $\begin{array}{c}\text { Cambio } \\
\mathrm{F}\end{array}$ \\
\hline $\begin{array}{l}\text { 1.Supervisió } \\
\mathrm{n}\end{array}$ & -.241 & -.188 & -.122 & -.132 & -.146 & .041 & .041 & $9.114^{*}$ \\
\hline $\begin{array}{l}\text { Familiar } \\
\text { Responsabl } \\
\mathrm{e}\end{array}$ & & -.205 & -.179 & -.118 & -.113 & .065 & .024 & $7.379^{*}$ \\
\hline $\begin{array}{l}\text { 3.Entreteni } \\
\text { miento1 }\end{array}$ & & & .479 & .368 & .271 & .369 & .304 & $40.962^{* *}$ \\
\hline $\begin{array}{l}\text { 4.Logro } 1 \\
\text { L. }\end{array}$ & & & & .709 & .605 & .428 & .059 & $39.162^{* *}$ \\
\hline 5.Evasión1 & & & & & .237 & .448 & .020 & $33.816^{* *}$ \\
\hline
\end{tabular}

Tabla 5. Análisis de regresión entre variables que miden el abuso de Internet en adolescentes de 12-13 años. Nota: $* p<.05 . * * p<.01$. 1. Dimensiones de gratificaciones de uso

\begin{tabular}{|l|c|c|c|c|c|c|c|}
\hline \multicolumn{7}{|c|}{14 años en adelante } \\
\hline & 1 & 2 & 3 & 4 & $R^{2}$ & $\Delta R^{2}$ & $\begin{array}{c}\text { Cambio } \\
\mathrm{F}\end{array}$ \\
\hline $\begin{array}{l}\text { 1.Uso } \\
\text { Responsable }\end{array}$ & -.490 & -.421 & -.440 & -.443 & .123 & .123 & $47.630^{* *}$ \\
\hline 2.Evasión1 & & .561 & .351 & .339 & .310 & .187 & $75.828^{* *}$ \\
\hline 3.Entretenimiento1 & & & .259 & .204 & .353 & .043 & $61.215^{* *}$ \\
\hline $\begin{array}{l}\text { 4.Autopresentación } \\
1\end{array}$ & & & & .287 & .364 & .011 & $48.085^{* *}$ \\
\hline
\end{tabular}

Tabla 6. Análisis de regresión entre variables que miden el abuso de Internet en adolescentes de 14 años en adelante. Nota: $* p<.05 . * * p<.01 . \quad 1$. Dimensiones de gratificaciones de uso

\section{Discusión y conclusiones}

El objetivo de este trabajo era analizar las relaciones entre el abuso de Internet y variables personales y contextuales no estudiadas con anterioridad de manera conjunta: las gratificaciones por las que los adolescentes usan Internet, el uso responsable o netiqueta que hacen en él y la supervisión familiar que reciben, analizados según el género y la edad de cara a establecer estrategias eficaces para un uso adaptado de la tecnología. En términos generales, los resultados apuntan a que las gratificaciones analizadas especialmente el entretenimiento y la evasión 
promueven el abuso y, sin embargo, el ser respetuoso con los demás se revela como un factor de protección junto a la supervisión familiar.

Al analizar las relaciones entre el abuso de Internet y las variables estudiadas -primer objetivo de esta investigación-, los resultados muestran que la relación con la supervisión familiar es significativa y negativa para las chicas y para los menores de 12-13 años. Estos datos confirman los hallados en investigaciones anteriores del contexto español (Díaz-López et al., 2020; Martínez et al., 2020). Esta misma tendencia se puede observar en otros países. Así en la mayoría de países europeos las chicas también son más acompañadas y orientadas con alguna excepción como Francia y Alemania donde lo hacen de forma similar en ambos géneros y en todos ellos, a medida que aumenta la edad disminuye la mediación familiar sobretodo en medidas restrictivas (Smahel et al., 2020). También en otros contextos como en Australia, la media de orientación y acompañamiento son más intensas en los más pequeños (Shin \& Lwin, 2017). Por otro lado, al analizar las relaciones del abuso de Internet con el uso responsable de los adolescentes al interaccionar en Internet, se concluye que, tanto para los chicos como las chicas de todas las edades estudiadas, la asociación entre ellas es significativa y negativa confirmando lo sugerido por Ortega et al., (2012). Con nuestros resultados podemos aportar que estas relaciones adquieren mayores puntuaciones a medida que tienen más edad y respecto al género, es en las chicas donde alcanza mayor asociación. Este dato nos lleva a reflexionar en la necesidad de mejorar la supervisión familiar y el uso responsable con los varones, ya que mediatizan en gran medida ciertas conductas sexistas como altos porcentajes de conductas poco adecuadas por el contexto cultural. Resultaría relevante incorporar orientaciones específicas para favorecer una educación respetuosa en el uso de Internet en los planes de igualdad y de convivencia de los centros educativos españoles.

Las relaciones encontradas entre el abuso de Internet y las gratificaciones buscadas en su uso indican que, en general, la evasión y el entretenimiento son las motivaciones más frecuentes, resultados coincidentes con otras investigaciones (Malo-Cerrato et al., 2018; Park \& Lee, 2014). También evidencian que las chicas puntúan más alto en evasión mientras que los chicos priorizan el entretenimiento. Estos resultados nos permiten comprender mejor a los adolescentes en Internet y podría estar en relación a lo sugerido por Twenge \& Martin, (2020) en el sentido de que las adolescentes pasan más tiempo en las redes sociales y los niños pasan más tiempo jugando. Por ello, la evasión podría explicar, en gran parte, la asociación encontrada por algunos autores entre el tiempo pasado en línea y el uso problemático de Internet en las chicas (Marino et al., 2018).

Nuestro segundo objetivo era analizar las variables predictoras del abuso de Internet. En el modelo explicativo obtenido para las chicas y chicos, la supervisión familiar resulta factor de protección de las chicas mientras que el uso responsable de Internet o netiqueta lo es para ambos géneros. Este resultado supone una relevante aportación pues hasta ahora la literatura ha identificado como factor de riesgo para el abuso la cantidad de tiempo que se utiliza Internet (Müller et al., 2017; Rial et al., 2014) y nuestros resultados indican que el modo de uso también influye en la promoción de este riesgo. Es decir, aquellos adolescentes que guardan la netiqueta mientras navegan e interactúan en Internet están más protegidos lo que nos indica una línea educativa para prevenir el abuso de internet.

Por otro lado, respecto a los factores de riesgo, en el caso de las chicas la búsqueda de evasión es la gratificación que más aporta en la predicción del abuso a Internet y en los chicos el entretenimiento. Esta situación nos lleva a reflexionar sobre la importancia de hacer conscientes a los adolescentes sobre la materialización en hábitos diarios de estas gratificaciones pues son éstos los que promueven el riesgo adictivo en mayor medida, más que uso social (Van Deursen et al., 2015). Conviene que tengan en cuenta que, acciones que carecen de riesgo como visitar con frecuencia el sitio de un youtuber o una red social sea para evadirse o entretenerse, al convertirse en actos automáticos los acerca al abuso de Internet. 
En el modelo predictivo según la edad, podemos concluir que los factores de protección, en los menores de 12 y 13 años, son la supervisión familiar y el uso responsable (netiqueta) y sus factores de riesgo la búsqueda del entretenimiento cómo gratificación principal seguido del logro. En el caso de los que tienen 14 años en adelante, la netiqueta es su único factor de protección, pero con un peso mayor respecto a los de 12-13 años, y las gratificaciones que más predicen el abuso de Internet son la evasión, la autopresentación y en menor medida, el entretenimiento. Una explicación a estos resultados puede ser que, en los más jóvenes, el uso mayoritario parece centrarse en el ocio y a medida que crecen, Internet se convierte en un instrumento para su autoestima social (Valkenburg et al., 2017), el sentido de pertenencia y la construcción de la identidad (Allen et al., 2014).

Los resultados han evidenciado que la supervisión familiar predice en menor medida que la netiqueta la prevención del abuso a Internet y sólo lo hace en el caso de las chicas y los menores de 12-13 años. Este hecho puede tener causas diversas. Una razón que puede estar detrás de estos datos es que, según un estudio realizado en 19 países europeos, España es uno de los países donde el nivel de mediación familiar tanto de acompañamiento como de control de tiempo y contenidos es de los más bajas de Europa. El 52\% de los encuestados entre 9 y 16 años reflejan que nunca o casi nunca los padres hablan sobre el uso o comparten actividades en línea con ellos frente al $17 \%$ Francia, $19 \%$ de Alemania y $26 \%$ de Italia (Smahel et al., 2020). Otros países como Suiza, Polonia y Chequia alcanzan niveles similares a España acercándose Noruega y Portugal, por lo que no cabe duda que la supervisión digital entraña numerosos retos y dificultades. Algunas de las dificultades con que los padres españoles se encuentran al tratar de ayudar a sus hijos a utilizar Internet son, entre otras, tener menor nivel de conocimiento del uso de Internet que sus hijos, saber negociar las normas e inseguridad sobre cómo enseñarles a vivir sin depender de la tecnología y saber qué hacer para controlar el acceso y lo que hacen en Internet (Bartau-Rojas et al., 2018). En esta línea, los padres suecos reflejan que cuando conocen las experiencias negativas de sus hijos es cuando aumenta su preocupación sobre estos temas (Sorbring, 2014). Esto pone de manifiesto la urgencia de proporcionar recursos y formación de la competencia digital de los padres tal como constata Gairín-Sallán \& Mercader, (2018) al evidenciar que más del 95\% de los padres españoles afirman necesitar formación sobre el uso de Internet de cara a la educación de sus hijos. En este sentido promover colaboración familia-escuela puede resultar especialmente útil por el papel destacado de la escuela en el desarrollo de la competencia digital del alumno y de la familia por la rapidez en cómo evolucionan las aplicaciones y dispositivos (Bartau-Rojas et al., 2018). Por otro lado, diversos estudios internacionales evidencian que una participación activa en línea con los niños, es decir, escuchar y dialogar sobre experiencias en línea es la forma en la que perciben los padres mayor satisfacción en la ayuda que prestan a sus hijos en Internet (Cheung, 2010) por ello junto a la formación parece necesario dotar a los padres de estrategias que les permitan conversar con sus hijos. Este tipo de supervisión familiar implica mayores desafíos en comparación con la mediación de los medios tradicionales entre otros motivos porque el uso de Internet se lleva a cabo en un entorno más privado que el presencial y "estar en línea" es mayoritariamente considerado como una actividad del entorno personal (Symons et al., 2017). Además, desde un enfoque evolutivo también requiere un apoyo emocional a las familias con adolescentes al ser una etapa de mayor autonomía y donde se suelen multiplicar los posibles conflictos intrafamiliares.

Por último y considerando los factores comunes a la edad y el género respecto al abuso de Internet podríamos establecer que los perfiles de mayor riesgo son los chicos de 12 y 13 años que buscan entretenerse como principal gratificación al usar Internet y respecto a los adolescentes de 14 años en adelante, el perfil de mayor riesgo serían las chicas que usan Internet para evadirse y tienen un bajo nivel de netiqueta en sus interacciones virtuales.

Estos resultados, en su conjunto, plantean implicaciones educativas importantes. El uso responsable ha resultado ser un factor de protección del abuso de Internet para todas las edades y 
para ambos géneros lo que evidencia la necesidad de incorporar, entre los adolescentes, la educación del aprendizaje de la netiqueta e ir más allá de la sensibilización sobre los riesgos cibernéticos respecto a su educación digital. En este sentido, complementando programas para mejorar la supervisión familiar, creemos relevante potenciar estructuras de apoyo a nivel del alumnado (Barrio et al., 2011) que colaboren en la sensibilización de un uso responsable de Internet y que posibilite sacar a la luz determinadas conductas nocivas e inapropiadas en las redes sociales, algunas de ellas relacionadas con el abuso y la violencia. Desde esta perspectiva, el empoderamiento y protagonismo del alumnado, el trabajo colaborativo en redes de centros, la implementación de programas interdisciplinares de ocio saludable que abarquen diferentes materias incluyendo la tutoría pueden ser aspectos preventivos y positivos para un uso responsable de Internet. Por otro lado, a la vista de las gratificaciones buscadas por los menores se hace necesaria programas formativos familiares para la educación del ocio para ayudarles a buscar en el entorno presencial las gratificaciones que encuentran en el entorno online (Masur et al., 2014) así como equilibrar el número de actividades online y offline (Lee, \& Han, 2010) con el reto de superar su posible aislamiento y reforzar la parentalidad positiva.

Como limitaciones de este estudio señalamos que los resultados se han obtenido con autoinformes y pueden tener sesgos de respuesta que podrían completarse, en futuros estudios, con información que aporten familias y docentes. También convendría realizar estos análisis en una muestra más amplia de menores. Además, como líneas futuras de trabajo podría ser útil analizar la eficacia de las distintas estrategias de mediación familiar y conocer las razones que llevan a los adolescentes a tales gratificaciones.

Al mismo tiempo, esta investigación realiza tres aportaciones novedosas. Por un lado, la necesidad de incorporar pautas educativas distintas sobre las gratificaciones de uso de Internet según el género y la edad. Por otro, el uso responsable (netiqueta) emerge como factor protector ante el abuso de Internet. Y, por último, algunas investigaciones han puesto de manifiesto la relación existente entre la dependencia de Internet y el ciberbullying (Yudes-Gómez et al., 2018) sin embargo, hasta el momento no se había evidenciado que las conductas relacionales respetuosas (netiqueta) influyen de manera positiva en el uso adaptativo de Internet.

\section{Agradecimientos}

Esta investigación tiene soporte del Grupo de Investigación Educaviva, la Cátedra de Inteligencia colectiva de la Universidad de Zaragoza y la cobertura de los contratos de Formación de Profesorado Universitario del Gobierno de Aragón.

\section{Referencias}

Allen, K. A., Ryan, T., Gray, D. L., McInerney, D. M., \& Waters, L. (2014). Social Media Use and Social Connectedness in Adolescents: The Positives and the Potential Pitfalls. The Australian Educational and Developmental Psychologist, 31(1), 18-31. https://doi.org/10.1017/ edp.2014.2

Ang, R. P., Huan, V. S., \& Florell, D. (2014). Understanding the Relationship Between Proactive and Reactive Aggression, and Cyberbullying Across United States and Singapore Adolescent Samples. Journal of Interpersonal Violence, 29(2), 237-254. https://doi.org/ $10.1177 / 0886260513505149$ 
Baldry, A. C., Sorrentino, A., \& Farrington, D. P. (2019). Cyberbullying and cybervictimization versus parental supervision, monitoring and control of adolescents' online activities. Children and Youth Services Review, 96, 302-307. https://doi.org/10.1016/j.childyouth.2018.11.058

Barrio, C., Barrios, A., Granizo, L., van der Meulen, K., Andrés, S. y Gutiérrez, H. (2011). Contribuyendo al bienestar emocional de los compañeros: evaluación del Programa Compañeros Ayudantes en un instituto madrile- ño. European Journal of Education and Psychology, 1(4), 5-17.

Bartau-Rojas, I., Aierbe-Barandiaran, A., \& Oregui-González, E. (2018). Parental mediation of the Internet use of Primary students: Beliefs, strategies and difficulties. Comunicar, 26(54), 7179. https://doi.org/10.3916/C54-2018-07

Berríos, L., Buxarrais, M. R., \& Garcés, M. S. (2015). ICT use and parental mediation perceived by Chilean children. Comunicar, 23(45), 161-168. https://doi.org/10.3916/C45-2015-17

Caplan, S. E. (2010). Theory and measurement of generalized problematic Internet use: A twostep approach. Computers in Human Behavior, 26(5), 1089-1097. https://doi.org/10.1016/ j.chb.2010.03.012

Casas, J. A., Ruiz-Olivares, R., \& Ortega-Ruiz, R. (2013). Validation of the internet and social networking experiences questionnaire in Spanish adolescents. International Journal of Clinical and Health Psychology, 13(1), 40-48. https://doi.org/10.1016/S1697-2600(13)70006-1

Chang, F.-C., Chiu, C.-H., Chen, P.-H., Chiang, J.-T., Miao, N.-F., Chuang, H.-Y., \& Liu, S. (2019). Children's use of mobile devices, smartphone addiction and parental mediation in Taiwan. Computers in Human Behavior, 93, 25-32. https://doi.org/10.1016/j.chb.2018.11.048

Chen, H.-T., \& Kim, Y. (2013). Problematic Use of Social Network Sites: The Interactive Relationship Between Gratifications Sought and Privacy Concerns. Cyberpsychology, Behavior, and Social Networking, 16(11), 806-812. https://doi.org/10.1089/cyber.2011.0608

Cheung, Y. (2010). Cyber-Parenting: Internet Benefits, Risks and Parenting Issues. Journal of Technology in Human Services, 28(4), 252-273. https://doi.org/ $10.1080 / 15228835.2011 .562629$

Díaz-López, A., Maquilón-Sánchez, J., \& Mirete-Ruiz, A. (2020). Uso desadaptativo de las TIC en adolescentes: Perfiles, supervisión y estrés tecnológico. Comunicar, 28(64), 29-38. https:// doi.org/DOI https://doi.org/10.3916/C64-2020-03

Durkee, T., Carli, V., Floderus, B., Wasserman, C., Sarchiapone, M., Apter, A., Balazs, J., Bobes, J., Brunner, R., Corcoran, P., Cosman, D., Haring, C., Hoven, C., Kaess, M., Kahn, J.-P., Nemes, B., Postuvan, V., Saiz, P., Värnik, P., \& Wasserman, D. (2016). Pathological Internet Use and RiskBehaviors among European Adolescents. International Journal of Environmental Research and Public Health, 13(3), 294. https://doi.org/10.3390/ijerph13030294

Fundación Mapfre. (2014). Tecnoadicción. Más de 70.000 adolescentes son tecnoadictos. Seguridad y Medioambiente, 1, 66-69.

Gairín-Sallán, J., \& Mercader, C. (2018). Usos y abusos de las TIC en los adolescents. Revista de Investigacion Educativa, 36(1), 125-140. https://doi.org/10.6018/rie.36.1.284001

García-Ruiz, R., Tirado Morueta, R., \& Hernando Gómez, A. (2018). Redes sociales y estudiantes: motivos de uso y gratificaciones. Evidencias para el aprendizaje. Aula Abierta, 47(3), 291. https://doi.org/10.17811/rifie.47.3.2018.291-298

Giménez, A. M., Luengo, J. A., \& Bartrina, M. J. (2017). ¿Qué hacen los menores en internet? Usos de las TIC, estrategias de supervisión parental y exposición a riesgos. Electronic Journal of 
Research in Education Psychology, 15(43), 533-552. https://doi.org/10.25115/ ejrep.43.16123

González-Vázquez, A., \& Igartua, J. J. (2018). ¿Por qué los adolescentes juegan videojuegos? Propuesta de una escala de motivos para jugar videojuegos a partir de la teoría de usos y gratificaciones. Cuadernos.Info, 42, 135-146. https://doi.org/10.7764/cdi.42.1314

Griffiths, M. D. (2010). The Role of Context in Online Gaming Excess and Addiction: Some Case Study Evidence. International Journal of Mental Health and Addiction, 8(1), 119-125. https:// doi.org/10.1007/s11469-009-9229-x

Heravi, A., Mubarak, S., \& Raymond Choo, K. K. (2018). Information privacy in online social networks: Uses and gratification perspective. Computers in Human Behavior, 84, 441-459. https://doi.org/10.1016/j.chb.2018.03.016

Igartua, J.-J., \& Rodríguez-de-Dios, I. (2016). Correlatos motivacionales del uso y la satisfacción con Facebook en jóvenes españoles. Cuadernos.Info, 38, 107-119. https://doi.org/10.7764/ cdi.38.848

INE (2020). Encuesta sobre equipamiento y uso de tecnologías de información y comunicación en los hogares. https://www.ine.es/dyngs/INEbase/es/operacion.htm? $\mathrm{c}=$ estadistica_C\&cid $=1254736176741 \&$ menu $=$ ultiDatos\&idp $=1254735976608$

Kaess, M., Durkee, T., Brunner, R., Carli, V., Parzer, P., Wasserman, C., Sarchiapone, M., Hoven, C., Apter, A., Balazs, J., Balint, M., Bobes, J., Cohen, R., Cosman, D., Cotter, P., Fischer, G., Floderus, B., Iosue, M., Haring, C., ... Wasserman, D. (2014). Pathological Internet use among European adolescents: psychopathology and self-destructive behaviours. European Child \& Adolescent Psychiatry, 23(11), 1093-1102. https://doi.org/10.1007/s00787-014-0562-7

Kalmus, V., Blinka, L., \& Ólafsson, K. (2015). Does It Matter What Mama Says: Evaluating the Role of Parental Mediation in European Adolescents' Excessive Internet Use. Children \& Society, 29(2), 122-133. https://doi.org/10.1111/chso.12020

Katz, E., Blumler, J. G. Y Gurevitch, M. (1974). The uses of mass communications: Current perspectives on gratifications research. Sage.

Katz, E., Haas, H., \& Gurevitch, M. (1973). On the Use of the Mass Media for Important Things. American Sociological Review, 38(2), 164. https://doi.org/10.2307/2094393

Khurana, A., Bleakley, A., Jordan, A. B., \& Romer, D. (2015). The Protective Effects of Parental Monitoring and Internet Restriction on Adolescents' Risk of Online Harassment. Journal of Youth and Adolescence, 44(5), 1039-1047. https://doi.org/10.1007/s10964-014-0242-4

Kim, J., \& Haridakis, P. M. (2009). The Role of Internet User Characteristics and Motives in Explaining Three Dimensions of Internet Addiction. Journal of Computer-Mediated Communication, 14(4), 988-1015. https://doi.org/10.1111/j.1083-6101.2009.01478.x

Kuss, D., Griffiths, M., Karila, L., \& Billieux, J. (2014). Internet Addiction: A Systematic Review of Epidemiological Research for the Last Decade. Current Pharmaceutical Design, 20(25), 40264052. https://doi.org/10.2174/13816128113199990617

Lee, J. and Han, K. H. (2010). Difference analysis of risk and protection factors for internet addiction between computer science gifted students and average students. Journal of Gifted/ Talented Education, 20, 1005-1026.

Li, W., O'Brien, J. E., Snyder, S. M., \& Howard, M. O. (2015). Characteristics of Internet Addiction/ Pathological Internet Use in U.S. University Students: A Qualitative-Method Investigation. PLOS ONE, 1O(2), e0117372. https://doi.org/10.1371/journal.pone.0117372 
Livingstone, S., Ólafsson, K., Helsper, E. J., Lupiáñez-Villanueva, F., Veltri, G. A., \& Folkvord, F. (2017). Maximizing Opportunities and Minimizing Risks for Children Online: The Role of Digital Skills in Emerging Strategies of Parental Mediation. Journal of Communication, 67(1), 82-105. https://doi.org/10.1111/jcom.12277

Malo-Cerrato, S., Martín-Perpiñá, M.-M., \& Viñas-Poch, F. (2018). Uso excesivo de redes sociales: Perfil psicosocial de adolescentes españoles. Comunicar, 26(56), 101-110. https://doi.org/ 10.3916/C56-2018-10

Marino, C., Gini, G., Vieno, A., \& Spada, M. M. (2018). A comprehensive meta-analysis on Problematic Facebook Use. Computers in Human Behavior, 83, 262-277. https://doi.org/ 10.1016/j.chb.2018.02.009

Marino, C., Vieno, A., Pastore, M., Albery, I. P., Frings, D., \& Spada, M. M. (2016). Modeling the contribution of personality, social identity and social norms to problematic Facebook use in adolescents. Addictive Behaviors, 63, 51-56. https://doi.org/10.1016/j.addbeh.2016.07.001

Martínez, D. G., Doctora, I. C., \& Social, D. D. C. (2020). Estrategias online de mediación parental en contextos familiares de España Online parental mediation strategies in family contexts of Spain. Comunicar, 65, 67-76.

Masur, P. K., Reinecke, L., Ziegele, M., \& Quiring, O. (2014). The interplay of intrinsic need satisfaction and Facebook specific motives in explaining addictive behavior on Facebook. Computers in Human Behavior, 39, 376-386. https://doi.org/10.1016/j.chb.2014.05.047

Mo, P. K. H., Chan, V. W. Y., Wang, X., \& Lau, J. T. F. (2020). Gender difference in the association between internet addiction, self-esteem and academic aspirations among adolescents: $A$ structural equation modelling. Computers \& Education, 155, 103921. https://doi.org/10.1016/ j.compedu.2020.103921

Müller, K.W., Dreier, M., Beutel, M.E., Duven, E., Giralt, S., \& Wölfling, K. (2017). A hidden type of Internet addiction? Intense and addictive use of social networking sites in adolescence. Computers in Human Behaviour, 55, 172-177. https://doi.org/https://doi.org/10.1016/ j.chb.2015.09.007

Ortega, R., Del Rey, R., \& Sánchez, V. (2012). Nuevas dimensiones de la convivencia escolar y juvenil. Ciberconducta y relaciones en la red: ciberconvivencia. Ministerio de Educación, Cultura y Deporte. Gobierno de España. https://doi.org/10.13140/2.1.3141.1520

Papacharissi, Z., \& Mendelsohn, A. (2011). Toward a new(er) sociability: Uses, gratifications and social capital on Facebook. In S. Papathanassopoulos (Ed.), Communication and society. Media perspectives for; topics and issues (pp. 212-230). Routledge.

Papacharissi, Z., \& Rubin, A. M. (2000). Predictors of Internet Use. Journal of Broadcasting \& Electronic Media, 44(2), 175-196. https://doi.org/10.1207/s15506878jobem4402_2

Park, N., \& Lee, S. (2014). College Students' Motivations for Facebook Use and Psychological Outcomes. Journal of Broadcasting \& Electronic Media, 58(4), 601-620. https://doi.org/ $\underline{10.1080 / 08838151.2014 .966355}$

Park, S., Na, E. Y., \& Kim, E. mee. (2014). The relationship between online activities, netiquette and cyberbullying. Children and Youth Services Review, 42, 74-81. https://doi.org/10.1016/ j.childyouth.2014.04.002

Rial, A., Golpe, S., Gómez, P., \& Barreiro, C. (2014). Variables asociadas al uso problemático de internet entre adolescentes. In Health and Addictions (Vol. 15, Issue 1).

Rodríguez Gómez, D., Castro Ceacero, D., \& Meneses, J. (2018). Usos problemáticos de las TIC 
entre jóvenes en su vida personal y escolar. Comunicar: Revista Científica Iberoamericana de Comunicación y Educación, 56, 91-100.

Ryan, T., Chester, A., Reece, J., \& Xenos, S. (2014). The uses and abuses of Facebook: A review of Facebook addiction. Journal of Behavioral Addictions, 3(3), 133-148. https://doi.org/10.1556/ JBA.3.2014.016

Sasson, H., \& Mesch, G. S. (2019). Parental Mediation. In The International Encyclopedia of Media Literacy (pp. 1-6). Wiley. https://doi.org/10.1002/9781118978238.ieml0177

Shea. (1994). Netiquette. Albion Books.

Sherry J. L., Lucas, K., Greenberg, B. S., \& Lachlan, K. (2006). Video game uses and gratifications as predictors of use and game preference. In P. Vorderer \& J. Bryant (Ed.), Playing video games, motives, responses and consequences. Lawrence Erlabum Associates.

Shin, W., \& Kang, H. (2016). Adolescents' privacy concerns and information disclosure online: The role of parents and the Internet. Computers in Human Behavior, 54, 114-123. https:// doi.org/10.1016/j.chb.2015.07.062

Shin, W., \& Lwin, M. O. (2017). How does "talking about the Internet with others" affect teenagers' experience of online risks? The role of active mediation by parents, peers, and school teachers. New Media \& Society, 19(7), 1109-1126. https://doi.org/ $10.1177 / 1461444815626612$

Smahel, D., Zlamal, R., Machackova, HanaAbramczuk, K., Ólafsson, K., \& Staksrud, E. (2020). EU Kids Online 2020: Technical report. 1-47. https://doi.org/10.21953/Ise.47fdeqj01ofo

Sorbring, E. (2014). Parents' Concerns About Their Teenage Children's Internet Use. Journal of Family Issues, 35(1), 75-96. https://doi.org/10.1177/0192513X12467754

Symons, K., Ponnet, K., Walrave, M., \& Heirman, W. (2017). A qualitative study into parental mediation of adolescents' internet use. Computers in Human Behavior, 73, 423-432. https:// doi.org/10.1016/j.chb.2017.04.004

Twenge, J. M., \& Martin, G. N. (2020). Gender differences in associations between digital media use and psychological well-being: Evidence from three large datasets. Journal of Adolescence, 79, 91-102. https://doi.org/10.1016/j.adolescence.2019.12.018

Valkenburg, P. M., Koutamanis, M., \& Vossen, H. G. M. (2017). The concurrent and longitudinal relationships between adolescents' use of social network sites and their social self-esteem. Computers in Human Behavior, 76, 35-41. https://doi.org/10.1016/j.chb.2017.07.008

Van Deursen, A. J. A. M., Bolle, C. L., Hegner, S. M., \& Kommers, P. A. M. (2015). Modeling habitual and addictive smartphone behavior: The role of smartphone usage types, emotional intelligence, social stress, self-regulation, age, and gender. Computers in Human Behavior, 45, 411-420. https://doi.org/10.1016/j.chb.2014.12.039

Wang, Y., Wu, A. M. S., \& Lau, J. T. F. (2016). The health belief model and number of peers with internet addiction as inter-related factors of Internet addiction among secondary school students in Hong Kong. BMC Public Health, 16(1), 272. https://doi.org/10.1186/ s12889-016-2947-7

Yudes-Gómez, C., Baridon Chauvie, D., \& González-Cabrera, J. (2018). Cyberbullying and problematic Internet use in Colombia, Uruguay and Spain: Cross-cultural study. Comunicar, 56, 49-58. https://doi.org/10.3916/C56-2018-05 